\title{
SMS Text Messages for Parents for the Prevention of Child Drowning in Bangladesh: Acceptability Study
}

\author{
Md Mosharaf Hossain ${ }^{1 *}, \mathrm{PhD}$; Kulanthayan Mani ${ }^{2}$, PhD; Ruhani Mat Min ${ }^{1}, \mathrm{PhD}$ \\ ${ }^{1}$ Faculty of Business, Economics and Social Development, Universiti Malaysia Terengganu, Kuala Nerus, Terengganu, Malaysia \\ ${ }^{2}$ Safe Kids Malaysia and Department of Community Health, Faculty of Medicine and Health Sciences, Universiti Putra Malaysia, Selangor, Malaysia \\ *these authors contributed equally
}

\section{Corresponding Author:}

Kulanthayan Mani, PhD

Safe Kids Malaysia and Department of Community Health

Faculty of Medicine and Health Sciences

Universiti Putra Malaysia

43400 Serdang

Selangor

Malaysia

Phone: 600397692398

Email: kulan@upm.edu.my

\begin{abstract}
Background: In many cases, greater use is being made of mobile phone text messages as a means of communication between patients and health care providers in countries around the world.

Objective: We studied the use of mobile phones and the factors related to the acceptability of text messages for parents for the prevention of child drowning in Bangladesh.

Methods: From a randomized controlled trial involving 800 parents, 10\% (80/800) were selected, and socioeconomic status, mobile phone use, and acceptability of SMS text messages for drowning prevention were measured. Participants with at least one child under 5 years of age were selected from rural areas in Rajshahi District in Bangladesh. Mobile phone-based SMS text messages were sent to the participants. Multivariate regression was used to determine the factors related to the acceptability of text messages for the prevention of child drowning in Bangladesh.

Results: The acceptability of SMS text messages for the prevention of child drowning in Bangladesh was significantly lower among women (odds ratio [OR] $0.50,95 \%$ CI $0.12-1.96, P=.02)$ than among men, lower for parents older than 30 years $(\mathrm{OR}$ $0.17,95 \%$ CI $0.14-1.70, P=.01)$ compared to parents younger than 30 years, higher among parents who had an education $(\mathrm{OR}$ $1.63,95 \%$ CI 1.11-5.80, $P=.04$ ) than among illiterate parents, and higher among parents with a monthly household income over 7000 Bangladeshi Taka (approximately US \$82.54; OR 1.27, 95\% CI 1.06-1.96, $P=.05$ ) than among parents whose monthly income was less than 7000 Bangladeshi Taka.
\end{abstract}

Conclusions: The high percentage of mobile phone use and the acceptability of SMS text messages for parents for the prevention of child drowning are encouraging, in terms of identifying the best strategy for using such technologies, and deserve further evaluation.

(JMIR Mhealth Uhealth 2020;8(9):e16958) doi: $\underline{10.2196 / 16958}$

\section{KEYWORDS}

acceptability; SMS; drowning; parents

\section{Introduction}

Water plays an important role in children's daily life. They like to play with water, have fun, and sometimes are more adventurous. Children are always excited around water, no matter whether it is a pond, pool, lake, in an open field, or simply on or beside the road after rain falls. It is impossible for children to grow up without water, as besides playing, they need it to clean themselves, and they gain comfort and are cooled by it. Although water is considered to be an important element for children to survive, it is also a hazard if there is a lack of awareness concerning the dangers it presents [1]. 
A small child can drown in only a few inches of water-under a bucket, in a field, or in the bathtub. Drowning injuries have an epidemiological pattern. However, the pattern changes depending on the type of water body, age group, and activity. Drowning ranks among the top 3 causes of accidental deaths in most countries around the world, and the mortality rates are highest in children under 5 years of age [1].

Drowning is the leading cause of death for children aged 1 year and over in Bangladesh. According to the rate reported in the Bangladesh Health and Injury Survey [2], nearly 17,000 children drowned in 2004, approximately 46 per day, and close to 4 times more than the rate in other low-income countries [2]. This equates to approximately 188 children drowning a day. In Bangladesh, drowning rates are 10 to 20 times higher than those in high-income countries [2]. There is a question concerning whether drowning is always fatal or whether it is possible to survive. While many people think that drowning is always a fatal event, others think that near drowning is a result that is never lethal [2].

As a result, drowning injuries are now the leading cause of death, disability, and severe morbidity among children in lowand middle-income countries in Asia, such as Bangladesh. This compromises the gains that were previously achieved, at high cost, to prevent other causes of illness and injury among children, and it jeopardizes the continued progress in survival and security. One conclusion is that drowning injuries are so important that they must be treated first [3].

Drowning reduces the impact of other interventions for children, as children who died from drowning often received conventional vaccines, vitamin A supplementation, and other food aid. They often used early development and education programs, and such investments are lost when a child drowns. Effective drowning prevention interventions are available at all stages of childhood and are cost-effective alongside traditional interventions for children [2].

Mobile phones and SMS text messages are gradually being used between patients and their health care providers in many countries around the world because of their communication potential [4]. Several studies [5-7] have shown that SMS text message-based counseling and observation can restore patient behavior and health outcomes. However, the potential use of SMS text messages in clinical settings in low-income countries has not been well established. The feasibility of implementing such technologies is not clearly demonstrated, nor is there a cost-effective and sustainable payment mechanism or commercial model that can be improved [8-12]. Information concerning how patients appreciate or perceive the use of SMS text messages from a mobile phone to improve disease management can be valuable in implementing such methods in primary care. Therefore, this study aimed to determine the access to mobile phones and the willingness to receive SMS text messages on a mobile phone; and determine the factors related to the willingness to receive mobile phone-based SMS text messages concerning child drowning prevention.

\section{Methods}

\section{Overview}

The researchers focused on the acceptability of text messages for child drowning prevention as part of a randomized controlled trial (International Standard Randomized Controlled Trial Number; ISRCTN13774693) of a mobile phone-based SMS text messages intervention aimed at improving the knowledge, attitude, and practices of parents about child drowning in Bangladesh. As part of a larger study, the design of the study and the data collection procedure followed the protocol of a previously published randomized controlled trial [13]. The sample size was 80 parents from 2 villages (10\% of the randomized controlled trial's sample size). The parents received an SMS text message before the interview.

The mobile phone-based SMS text message intervention aimed to improve parents' knowledge, attitudes, and practices in relation to the prevention of child drowning in Bangladesh. The researcher developed the SMS text messages based on focus group discussions and literature reviews. The messages used informal language and were sent every Friday. On Friday morning, each week, the research team sent out SMS text messages to parents in the intervention group. The message was typically 150-200 characters long and in the Bangla language.

Data were collected through face-to-face interviews in rural areas using a structured questionnaire, which was conducted by a team of 2 qualified research assistants. The inclusion criteria were the same as those for the published study protocol [13]. Parents meeting the selection criteria were included in the study. The questionnaires included questions on socioeconomic characteristics (age, sex, marital status, education, and income), mobile phone use, SMS text message use, and drowning knowledge.

\section{Data Analysis}

The researchers performed chi-square tests to assess the importance of the responses and associated factors regarding SMS text messaging concerning drowning in childhood. A univariate logistic regression analysis was used to evaluate the relationship between SMS text message reading and the individual variables. Factors having a statistically significant association with the reading of text messages on child drowning in univariate analysis $(P$ value $<.25)$ were included in the final logistic model $(P$ value $<.05)$ taking into account other variables. SPSS statistical software (version 22; IBM Corp) was used for data analysis. A value of $P<.05$ was considered statistically significant.

\section{Ethical Statement}

The ethical approval for this study was obtained from University Putra Malaysia (UPM/TNCPI/RMC/1.4.18.1 (JKEUPM)/F2) and the Centre for Injury Prevention and Research, Bangladesh. Informed written agreements were obtained from all the respondents before the data were collected. All the respondents were assured that the data would only be used for research purposes and that all the answers would be kept confidential. 


\section{Results}

The association between the acceptability of text messages about childhood drowning and the selected sociodemographic characteristics of parents are presented in Table 1. Table 1 reveals the associations between parent age, gender, educational status, occupation, monthly income, the ability to read SMS text messages, liking SMS text messages, and having Bangla language on their phone with the acceptability of text messages on childhood drowning. The acceptability of text messages concerning childhood drowning prevention was significantly higher among males $\left(76 \%, \chi^{2}=4.39, P<.001\right)$, parents aged less than 30 years $\left(82 \%, \chi^{2}=6.54, P<.001\right)$, literate parents $(76 \%$, $\left.\chi^{2}=4.98, P<.001\right)$, parents with a monthly income of more than 7000 BDT $\left(80 \%, \chi^{2}=5.69, P<.001\right)$, parents who had the ability to read an SMS text messages $\left(71 \%, \chi^{2}=3.94, P<.001\right)$, parents who liked mobile phone-based SMS text messages (74\%, $\left.\chi^{2}=7.51, P<.001\right)$, and parents who had Bangla on the mobile phone $\left(73 \%, \chi^{2}=5.63, P<.001\right)$ (Table 1). There were no significant associations between parents' occupation, type of mobile phone, use of mobile phone, knowledge about drowning with the acceptability of text messages on childhood drowning. 
Table 1. The results of chi-square tests concerning the acceptability of text messages about childhood drowning among various sociodemographic variables in Bangladesh.

\begin{tabular}{|c|c|c|c|c|c|}
\hline \multirow[t]{2}{*}{ Variables } & \multirow[t]{2}{*}{ All $(\mathrm{N}=80), \mathrm{n}(\%)$} & \multicolumn{2}{|l|}{ Acceptability } & \multicolumn{2}{|l|}{ Comparison } \\
\hline & & No $(n=26), n(\%)$ & Yes $(\mathrm{n}=54), \mathrm{n}(\%)$ & Chi-square $(d f)$ & $P$ value \\
\hline Age (years) & & & & $6.54(1,80)$ & $<.001$ \\
\hline Below 30 & $38(47)$ & $7(18)$ & $31(82)$ & & \\
\hline More than 30 & $42(53)$ & $19(45)$ & $23(55)$ & & \\
\hline Gender & & & & $4.39(1,80)$ & $<.001$ \\
\hline Male & $50(63)$ & $12(24)$ & $38(76)$ & & \\
\hline Female & $30(37)$ & $14(47)$ & $16(53)$ & & \\
\hline Educational status & & & & $4.98(1,80)$ & $<.001$ \\
\hline Illiterate & $30(37)$ & $14(47)$ & $16(53)$ & & \\
\hline Literate & $50(63)$ & $12(24)$ & $38(76)$ & & \\
\hline Occupation & & & & $0.97(1,80)$ & .563 \\
\hline Housewife/Farmer & $66(83)$ & $21(38)$ & $38(62)$ & & \\
\hline Other & $14(17)$ & $5(17)$ & $16(83)$ & & \\
\hline Monthly income (BDT ${ }^{\mathbf{a}}$ ) & & & & $5.69(1,80)$ & .001 \\
\hline Less than 7000 & $40(50)$ & $8(20)$ & $22(55)$ & & \\
\hline More than 7000 & $40(50)$ & $18(45)$ & $32(80)$ & & \\
\hline Phone type & & & & $0.38(1,80)$ & .781 \\
\hline Normal & $68(85)$ & $11(37)$ & $19(63)$ & & \\
\hline Smartphone & $12(15)$ & $15(30)$ & $35(70)$ & & \\
\hline Phone use & & & & $0.36(1,80)$ & .144 \\
\hline Father & $30(37)$ & $21(38)$ & $45(66)$ & & \\
\hline Mother & $50(63)$ & $5(29)$ & $9(71)$ & & \\
\hline Ability to read SMS text messages & & & & $3.94(1,80)$ & $<.001$ \\
\hline No & $10(12)$ & $6(50)$ & $4(50)$ & & \\
\hline Yes & $70(88)$ & $20(29)$ & $50(71)$ & & \\
\hline Liked SMS text messages & & & & $7.51(1,80)$ & $<.001$ \\
\hline No & $12(15)$ & $8(66)$ & $4(34)$ & & \\
\hline Yes & $68(85)$ & $18(26)$ & $50(74)$ & & \\
\hline Have Bangla language on phone & & & & $5.63(1,80)$ & $<.001$ \\
\hline No & $11(14)$ & $7(63)$ & $4(37)$ & & \\
\hline Yes & $69(86)$ & $19(27)$ & $50(73)$ & & \\
\hline Knowledge about drowning & & & & $3.94(1,80)$ & .346 \\
\hline No & $10(12)$ & $6(50)$ & $4(50)$ & & \\
\hline Yes & $70(88)$ & $20(29)$ & $50(71)$ & & \\
\hline
\end{tabular}

${ }^{\mathrm{a}} \mathrm{BDT}$ : Bangladeshi Taka; an exchange rate of US \$1 to 0.012 BDT is applicable.

In the logistic analysis (Table 2), the acceptability of text messages on childhood drowning was significantly higher among males. The odds ratio (OR) of 0.50 for females was less than 1 , indicating that female respondents considered text messages on childhood drowning to be 0.50 (adjusted OR 0.50, $P=.02,95 \%$ CI $0.12-1.96$ ) times less acceptable than their male counterparts.Parents aged more than 30 years considered text messages on childhood drowning to be 0.17 times less acceptable than parents aged less than 30 years (OR 0.17, $P=.01$, 95\% CI 0.14-1.70). Parents who had an education considered text messages on childhood drowning to be 1.63 times (OR $1.63, P=.04,95 \%$ CI $1.11-5.80)$ less acceptable than parents who had no education, those with a household income of more than 7000 BDT considered text messages on childhood 
drowning to be 1.27 times more acceptable than those with a household income of less than 7000 BDT (OR 1.27, $P=.05$, 95\% CI 1.06-1.96), and parents who had Bangla on their mobile phone considered text messages on childhood drowning to be 6.46 times more acceptable than parents who did not have the Bangla language on their mobile phone (OR 6.46, $P=.05,95 \%$
CI 1.89-6.65) (Figure 1). There were no significant associations among parents' occupation (OR 7.92, $P=.052$, 95\% CI 0.97-64.33) or knowledge about drowning (OR 3.73, $P=.05$, 95\% CI 0.95-14.71) with the acceptability of text messages on childhood drowning.

Table 2. Results of the logistic analysis of the acceptability of text messages on childhood drowning among various sociodemographic variables.

\begin{tabular}{|c|c|c|c|c|c|c|}
\hline Variable $^{\mathrm{a}}$ & $\mathrm{OR}^{\mathrm{b}}$ & $P$ value & $95 \% \mathrm{CI}$ & Adjusted OR & $P$ value & $95 \% \mathrm{CI}$ \\
\hline \multicolumn{7}{|l|}{ Age (years) } \\
\hline Below $30^{\mathrm{c}}$ & 1 & - & - & 1 & - & - \\
\hline More than 30 & 0.27 & $<.001$ & $0.09-0.75$ & 0.17 & $<.001$ & $0.14-1.70$ \\
\hline \multicolumn{7}{|l|}{ Gender } \\
\hline Male $^{\mathrm{c}}$ & 1 & - & - & 1 & - & - \\
\hline Female & 0.36 & .06 & $0.13-0.94$ & 0.5 & .02 & $0.12-1.96$ \\
\hline \multicolumn{7}{|l|}{ Educational status } \\
\hline Illiterate $^{\mathrm{c}}$ & 1 & - & - & 1 & - & - \\
\hline Literate & 2.77 & .03 & $1.05-7.29$ & 1.63 & .04 & $1.11-5.80$ \\
\hline \multicolumn{7}{|l|}{ Occupation } \\
\hline Housewife/Farmer ${ }^{\mathrm{c}}$ & 1 & - & - & 1 & - & - \\
\hline Other & 7.92 & 0.052 & $0.97-64.33$ & 1.73 & .16 & $1.71-8.87$ \\
\hline \multicolumn{7}{|l|}{ Monthly income (BDT ${ }^{\mathbf{d}}$ ) } \\
\hline Less than $7000^{\mathrm{c}}$ & 1 & - & - & 1 & - & - \\
\hline More than 7000 & 1.30 & $<.001$ & $0.98-1.82$ & 1.27 & .06 & $1.06-1.96$ \\
\hline \multicolumn{7}{|c|}{ Ability to read SMS text messages } \\
\hline $\mathrm{No}^{\mathrm{c}}$ & 1 & - & - & 1 & - & - \\
\hline Yes & 3.75 & .05 & $0.95-14.71$ & 1.04 & .98 & $0.08-12.91$ \\
\hline \multicolumn{7}{|l|}{ Liked SMS text messages } \\
\hline $\mathrm{No}^{\mathrm{c}}$ & 1 & - & - & 1 & - & - \\
\hline Yes & 5.55 & $<.001$ & $1.49-20.70$ & 1.38 & .78 & $0.13-13.87$ \\
\hline \multicolumn{7}{|c|}{ Have Bangla language on phone } \\
\hline $\mathrm{No}^{\mathrm{c}}$ & 1 & - & - & 1 & - & - \\
\hline Yes & 4.6 & .02 & $1.20-17.53$ & 6.46 & .053 & $1.89-6.65$ \\
\hline \multicolumn{7}{|c|}{ Knowledge about drowning } \\
\hline $\mathrm{No}^{\mathrm{c}}$ & 1 & - & - & 1 & - & - \\
\hline Yes & 3.73 & .05 & $0.95-14.71$ & 1.26 & .99 & $0.10-9.30$ \\
\hline
\end{tabular}

\footnotetext{
${ }^{\text {a }}$ Model Summary-2-loglikelihood: 71.12; Cox \& Snell R Square: 0.31; Negelikereke R: 0.43; model chi-square: 29.77.

${ }^{\mathrm{b}} \mathrm{OR}$ : odds ratio.

${ }^{\mathrm{c}}$ Reference.

${ }^{\mathrm{d}}$ BDT: Bangladeshi Taka; an exchange rate of US $\$ 1$ to 0.012 BDT is applicable.
} 
Figure 1. Forest plot of acceptability of text messages for parents on childhood drowning prevention odds ratios.

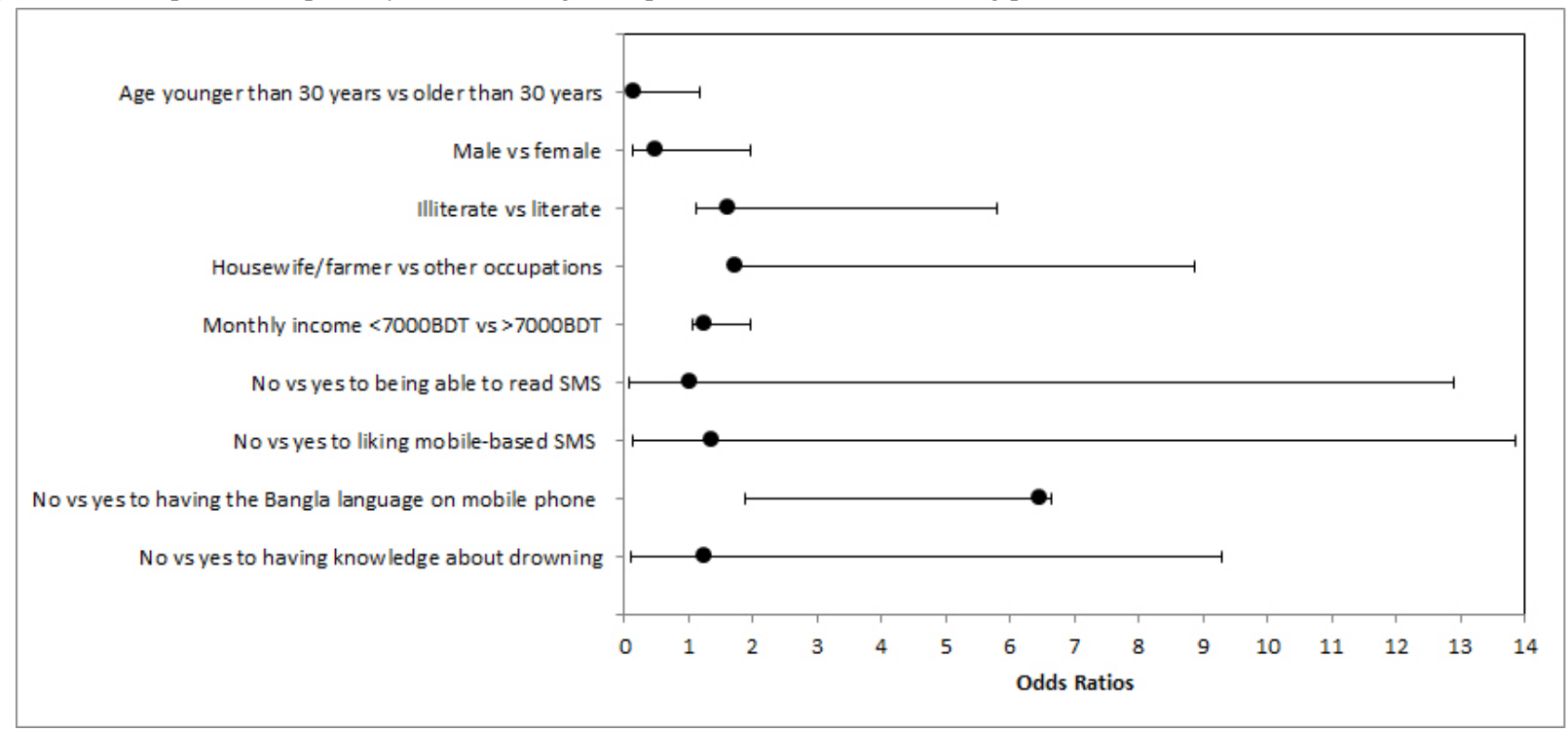

\section{Discussion}

\section{Principal Findings}

The aim of this research was to identify access to mobile phones, the willingness to receive mobile phone-based SMS text messages in relation to the prevention of childhood drowning, and associated factors among parents of children under 5 in the rural areas of Bangladesh. The results of this study show that there is high $(54 / 80,68 \%)$ mobile phone access among parents with children less than 5 years of age. Of the respondents who had access to mobile phones, the majority $(59 / 80,74 \%)$ would like to receive phone-based SMS text messages. This is similar to the findings of a study conducted in northwest Ethiopia concerning mobile health services among patients with diabetes by Jemere et al [14] in which $71 \%$ were willing to receive mobile phone-based health services.

Age, gender, educational status, monthly income, ability to read SMS text messages, liking mobile phone-based SMS text messages, and having Bangla language software on their mobile phone were associated with the acceptability of text messages on childhood drowning prevention in Bangladesh. The majority $(58 / 80,72 \%)$ were able to read SMS text messages. In the study, those in the age group of less than 30 years showed a higher acceptability of receiving text messages on childhood drowning than those in the other age group ( $\geq 30$ years); the acceptability of text messages on childhood drowning decreased slightly with age. Older respondents were less likely to possess the technology and knowledge compared to their younger counterparts, which could be the reason for the lower acceptability of text messages concerning childhood drowning. This is similar to the findings of a study in Bangladesh conducted by Islam et al [15] in which all the participants owned a mobile phone and about half of the participants reported being able to retrieve and read SMS text messages.

Among males, only those with a household income above 7000 BDT, and parents who had the Bangla language on their mobile phone were associated with the acceptability of text messages on childhood drowning prevention. An increase in education is associated with an increase in the proportion of income earners per household and had an impact on the acceptability of text messages on childhood drowning prevention, which is contrary to the assertions in the literature in this regard [15]. Those with a relatively higher income had greater willingness to read text messages on childhood drowning prevention, which is aligned with the expectations [15]. The association is likely to be due to the fact that a higher educational status is likely to lead to improved knowledge and awareness of drowning, and better access to mobile phone and mobile phone networks. Awareness concerning how to apply these new technologies to consumer health and enable patients to take control and play an active role in managing their health has increased. Consumer health interventions have been used, for example, to help people monitor their own health [16], provide social information and support, and remotely monitor their home [17]. Many studies [18-20] have used mobile phone technology for public health issues and have commented on other considerations for older people in terms of design, usability and functionality. Studies [18] have shown that interventions need to take into account psychological assumptions or barriers for use among older people.

Kotani et al [19] noted the success of using mobile phones to take pictures, even when older people refused to take pictures with traditional cameras. Mobile phones and telephones were perceived as informal and ubiquitous and were therefore considered acceptable while traditional cameras were not [20].

Mobile phone interventions for the health of the elderly are in their infancy and are just beginning to develop. The rapid development of mobile phones, coupled with the rapid aging of the population, provides an excellent opportunity to use mobile phone technology to better manage the health of seniors and positively impact their quality of life and well-being. Early childhood research in the field allow interested researchers to conduct research in all directions while pursuing the same goal of improving the lives of older people $[21,22]$. The prevalence of mobile phones, text messages, and in particular, the growing use of instant messengers and social networking apps in health care highlight ways to significantly integrate health care into 
users' daily activities instead of artificially complementing health care processes [23].

There are clear benefits from these interventions, such as further educating parents about their treatment, providing more opportunities for health care professionals and parents to interact efficiently and effectively, and improving health literacy in general. Directed and monitored interventions also provide end users with reliable information in a condensed and easily accessible form. More research is required to optimize the use of these interventions, with attention particularly focused on sensitive areas, such as cost and time burden for the providers and consumers of mobile phone health care services. The study participants in this trial mostly had (more than $87 \%$ ) access to a mobile phone, which might be different from other populations in Bangladesh [24-27].

\section{Strengths and Limitations}

This study provides quality evidence and establishes an association between the intervention and outcomes of the study in Bangladesh among parents of children aged under 5 years old.

These findings are consistent with the results reported by several researchers in similar studies [13-15] around the world concerning different injuries and provide information that is critical for controlling the drowning epidemic, especially in low- and middle-income countries that are highly burdened by such events.

The mobile phone intervention used in this study appears to be a relatively cheap and acceptable strategy for improving the drowning prevention knowledge, attitude, and practices of parents of children under 5 years old. The data concerning the cost-effectiveness of this strategy in comparison to those of other interventions were not reported because it was beyond the scope of this study.

\section{Conclusion}

The results of this study show that the vast majority of parents in rural areas in Bangladesh find SMS text messages to prevent drowning acceptable. Given that the number of drowning deaths is still high and the low cost of mobile phones in Bangladesh, a self-sustaining business model in low-income countries and other middle-income countries is possible. The age of the respondents, gender, educational status, monthly income, ability to read SMS text messages, liking mobile phone-based SMS text messages, and Bangla language software on the mobile phone were associated with the acceptance of text messages for preventing drowning. Based on this outcome, mobile phone SMS text messages implementation, such as self-monitoring, behavioral counseling, and interventions can be used to improve knowledge, attitudes, and practices.

\section{Authors' Contributions}

$\mathrm{KM}, \mathrm{MMH}$, and RMM wrote and approved the final manuscript. KM and MMH conceptualized and designed the research. KM and $\mathrm{MMH}$ undertook the formal data analysis and are responsible for all analysis. All authors critically revised this manuscript.

\section{Conflicts of Interest}

None declared.

\section{References}

1. Peden M. , Oyegbite K. Ozanne-Smith J World report on child injury prevention. URL: https://apps.who.int/iris/bitstream/ handle/10665/43851/9789241563574 eng.pdf;jsessionid=664C97FA1988A066A851587670D05633? sequence=1

2. Bangladesh Health and Injury Survey. Bangladesh Health and Injury Survey. Dhaka, Bangladesh: Government of the People's Republic of Bangladesh; 2005.

3. Rahman A. Bangladesh Health and Injury Survey: Report on Children. Dhaka, Bangladesh: Government of the People's Republic of Bangladesh, ICMH, UNICEF and TASC; 2005.

4. Chai SJ, Tan F, Ji Y, Wei X, Li R, Frost M. Community-Level Text Messaging for 2009 H1N1 Prevention in China. American Journal of Preventive Medicine 2013 Aug;45(2):190-196. [doi: 10.1016/j.amepre.2013.03.014]

5. Beratarrechea A, Lee AG, Willner JM, Jahangir E, Ciapponi A, Rubinstein A. The impact of mobile health interventions on chronic disease outcomes in developing countries: a systematic review. Telemed J E Health 2014 Jan;20(1):75-82 [FREE Full text] [doi: 10.1089/tmj.2012.0328] [Medline: 24205809]

6. Burner ER, Menchine MD, Kubicek K, Robles M, Arora S. Perceptions of successful cues to action and opportunities to augment behavioral triggers in diabetes self-management: qualitative analysis of a mobile intervention for low-income Latinos with diabetes. J Med Internet Res 2014;16(1):e25 [FREE Full text] [doi: 10.2196/jmir.2881] [Medline: 24476784]

7. Nhavoto JA, Grönlund A. Mobile technologies and geographic information systems to improve health care systems: a literature review. JMIR Mhealth Uhealth 2014;2(2):e21 [FREE Full text] [doi: 10.2196/mhealth.3216] [Medline: 25099368]

8. Brown S, Saint M. Value proposition for mhealth monitoring solution of diabetes. 2013 Presented at: 2013 IST-Africa Conference \& Exhibition; May 29-31, 2013; Nairobi, Kenya p. 1-11 URL: https://www.researchgate.net/publication/ 287396347 Value proposition for mhealth monitoring solution of diabetes

9. Okuboyejo S, Eyesan O. mHealth: Using Mobile Technology to Support Healthcare. OJPHI 2014 Feb 05;5(3). [doi: 10.5210/ojphi.v5i3.4865]

10. Bhuiyan T, Mahmud I, Alam B. Usability analysis of sms alert system for immunization in the context of Bangladesh. IJRET 2013 Nov 25;02(11):300-305. [doi: 10.15623/ijret.2013.0211046] 
11. Abebe N. Txt4Health: using mobile technology in public health communication and education campaigns. 2013 Presented at: National Conference on Health Communication, Marketing, and Media; 2013; Atlanta, GA.

12. Castaño PM, Stockwell MS, Malbon KM. Using digital technologies to improve treatment adherence. Clin Obstet Gynecol 2013 Sep;56(3):434-445. [doi: 10.1097/GRF.0b013e3182988a3b] [Medline: 23722918]

13. Hossain M, Mani KKC, Sidik SM, KS H, Fazlur Rahman A. Randomized controlled trial on drowning prevention for parents with children aged below five years in Bangladesh: a study protocol. BMC Public Health 2015 May 11;15(1). [doi: 10.1186/s12889-015-1823-1]

14. Jemere AT, Yeneneh YE, Tilahun B, Fritz F, Alemu S, Kebede M. Access to mobile phone and willingness to receive mHealth services among patients with diabetes in Northwest Ethiopia: a cross-sectional study. BMJ Open 2019 Jan 24;9(1):e021766. [doi: 10.1136/bmjopen-2018-021766]

15. Shariful ISM, Lechner A, Ferrari U, Seissler J, Holle R, Niessen LW. Mobile phone use and willingness to pay for SMS for diabetes in Bangladesh. J Public Health (Oxf) 2016 Mar;38(1):163-169. [doi: 10.1093/pubmed/fdv009] [Medline: 25687131]

16. Lam LT, Yang L, Liu Y, Geng WK, Liu DC. Different quantitative measures of the impact of injury deaths on the community in the Guangxi Province, China. Accid Anal Prev 2005 Jul;37(4):761-766. [doi: 10.1016/j.aap.2005.03.016] [Medline: 15949464]

17. Hossain M, Mani KKC, Sidik SM, Hayati KS, Rahman AKMF. Socio-demographic, environmental and caring risk factors for childhood drowning deaths in Bangladesh. BMC Pediatr 2015 Sep 10;15:114 [FREE Full text] [doi: 10.1186/s12887-015-0431-7] [Medline: 26357879]

18. Detmer D, Bloomrosen M, Raymond B, Tang P. Integrated personal health records: transformative tools for consumer-centric care. BMC Med Inform Decis Mak 2008;8:45 [FREE Full text] [doi: 10.1186/1472-6947-8-45] [Medline: 18837999]

19. Kotani K, Morii M, Asai Y, Sakane N. Application of mobile-phone cameras to home health care and welfare in the elderly: experience in a rural practice. Aust J Rural Health 2005 Jun;13(3):193-194. [doi: 10.1111/j.1440-1854.2005.00682.x] [Medline: 15932491]

20. Faucounau V, Riguet M, Orvoen G, Lacombe A, Rialle V, Extra J, et al. Electronic tracking system and wandering in Alzheimer's disease: a case study. Ann Phys Rehabil Med 2009;52(7-8):579-587 [FREE Full text] [doi: 10.1016/j.rehab.2009.07.034] [Medline: 19744906]

21. Joe J, Demiris G. Older adults and mobile phones for health: a review. J Biomed Inform 2013 Oct;46(5):947-954 [FREE Full text] [doi: 10.1016/j.jbi.2013.06.008] [Medline: 23810858]

22. Hossain MM, Mani KKC, Islam MR. Prevalence and determinants of the gender differentials risk factors of child deaths in Bangladesh: evidence from the Bangladesh demographic and health survey, 2011. PLoS Negl Trop Dis 2015 Mar;9(3):e0003616 [FREE Full text] [doi: 10.1371/journal.pntd.0003616] [Medline: 25747178]

23. Kamel E, Emam, SASameh E. Knowledge, attitude and practice among rural mothers about Home-related injuries in a rural area in El-Minia Governorate, Egypt. Science Journal of Public Health 2014;2(6):653-659. [doi:

10.11648/j.sjph.20140206.35]

24. Islam MZ. 87pc households use mobile. The Daily Star News. 2016 Jan 12. URL: https://www.thedailystar.net/frontpage/ $\underline{87 p c-h o u s e h o l d s-u s e-m o b i l e-200656}$

25. Tang S, Ghose B, Hoque MR, Hao G, Yaya S. Women Using Mobile Phones for Health Communication Are More Likely to Use Prenatal and Postnatal Services in Bangladesh: Cross-Sectional Study. JMIR Mhealth Uhealth 2019 Feb 28;7(2):e10645 [FREE Full text] [doi: 10.2196/10645] [Medline: 30816850]

26. Krinsky NI. Membrane antioxidants. Ann N Y Acad Sci 1988;551:17-32; discussion 32. [doi: 10.1111/j.1749-6632.1988.tb22317.x] [Medline: $\underline{3245661]}$

27. Hossain M, Mani KKC, Mohd Sidik S, Kadir Shahar H. The development of an intervention package to prevent children under five years old drowning in rural Bangladesh. Acta Paediatr 2016 Aug;105(8):e373-e378. [doi: 10.1111/apa.13447] [Medline: 27111395]

\section{Abbreviations}

OR: odds ratio 
Edited by G Eysenbach; submitted 07.11.19; peer-reviewed by D Ramesh, MA( Barry; comments to author 23.01.20; revised version received 24.02.20; accepted 23.03.20; published 23.09.20

Please cite as:

Hossain MM, Mani K, Mat Min R

SMS Text Messages for Parents for the Prevention of Child Drowning in Bangladesh: Acceptability Study JMIR Mhealth Uhealth 2020;8(9):e16958

URL: http://mhealth.jmir.org/2020/9/e16958/

doi: $\underline{10.2196 / 16958}$

PMID: $\underline{32965224}$

CMd Mosharaf Hossain, Kulanthayan Mani, Ruhani Mat Min. Originally published in JMIR mHealth and uHealth (http://mhealth.jmir.org), 23.09.2020. This is an open-access article distributed under the terms of the Creative Commons Attribution License (https://creativecommons.org/licenses/by/4.0/), which permits unrestricted use, distribution, and reproduction in any medium, provided the original work, first published in JMIR mHealth and uHealth, is properly cited. The complete bibliographic information, a link to the original publication on http://mhealth.jmir.org/, as well as this copyright and license information must be included. 